\title{
Temporal Specificity of Perceptual Learning in an Auditory Discrimination Task
}

\author{
Uma R. Karmarkar and Dean V. Buonomano ${ }^{1}$ \\ Departments of Neurobiology and Psychology, and the Brain Research Institute, University of California, Los Angeles, \\ Los Angeles, California 90095 USA
}

\begin{abstract}
Although temporal processing is used in a wide range of sensory and motor tasks, there is little evidence as to whether a single centralized clock or a distributed system underlies timing in the range of tens to hundreds of milliseconds. We investigated this question by studying whether learning on an auditory interval discrimination task generalizes across stimulus types, intervals, and frequencies. The degree to which improvements in timing carry over to different stimulus features constrains the neural mechanisms underlying timing. Human subjects trained on a $100-$ or $200-\mathrm{msec}$ interval discrimination task showed an improvement in temporal resolution. This learning generalized to a perceptually distinct duration stimulus, as well as to the trained interval presented with tones at untrained spectral frequencies. The improvement in performance did not generalize to untrained intervals. To determine if spectral generalization was dependent on the importance of frequency information in the task, subjects were simultaneously trained on two different intervals identified by frequency. As a whole, our results indicate that the brain uses circuits that are dedicated to specific time spans, and that each circuit processes stimuli across nontemporal stimulus features. The patterns of generalization additionally indicate that temporal learning does not rely on changes in early, subcortical processing, because the nontemporal features are encoded by different channels at early stages.
\end{abstract}

The auditory system is capable of temporal processing across a wide range of scales from microsecond timing, used in sound localization, up to several seconds (Jeffress 1948; Carr 1993; Buonomano and Karmarkar 2002). Timing in the range of tens to hundreds of milliseconds is a fundamental part of a wide range of sensory and motor tasks (Ivry 1996; Gibbon et al. 1997; Buonomano and Karmarkar 2002). In the auditory system, this range is used in the ability to discriminate the order, interval, and duration of sounds, which is important for speech processing (Shannon et al. 1995; Doupe and Kuhl 1999). Indeed, it is thought that deficits in this range of temporal processing may contribute to some language-based learning disabilities (Merzenich et al. 1996; Tallal et al. 1996). However, even for simple interval discrimination tasks, little is known about the neural mechanisms and areas involved in timing.

One unanswered question regarding temporal processing relates to the degree of its specificity for nontemporal features. For example, if a tone sounds at a regular interval, would the brain measure the interval with the same circuitry if the pitch of the tone was different? The answer would be yes if timing is centralized, meaning that temporal processing across qualitatively different tasks within or even across modalities is all computed by the same neural mechanisms. Alternatively, timing could be distributed,

${ }^{1}$ Corresponding author.

E-MAIL dbuono@ucla.edu; FAX (310) 825-2224.

Article and publication are at http://www.learnmem.org/cgi/doi/ $10.1101 / \mathrm{lm} .55503$ meaning that each perceptually different task requires its own circuitry. A second type of specificity concerns the task's temporal features themselves. A single 'universal clock' could be the sole timing mechanism for all intervals and/or durations, or there could be a set of dedicated circuits, each specific to given lengths of time (also called interval-based mechanisms; Ivry 1996).

It is possible to address these issues by examining the specificity of perceptual learning on an auditory temporal discrimination task. Previous studies have shown that auditory interval discrimination tasks undergo perceptual learning after training (Wright et al. 1997; Nagarajan et al. 1998; Westheimer 1999). Typically, the stimuli in these experiments consist of two brief tones separated by either a standard interval $(\mathrm{T})$ or a longer comparison interval equivalent to the standard plus a value $\Delta \mathrm{t}$. The subject is presented with both the standard and comparison interval and asked to judge whether the longer interval occurred first or second. Training over several days on a particular interval leads to improved performance as measured by a decreased, more accurate discrimination threshold. These experiments have shown that learning generalizes across untrained frequencies (the spatial domain) but not across intervals (the temporal domain; Wright et al. 1997). Other studies have indicated that improvements in interval discrimination generalize across sensory modalities (Nagarajan et al. 1998; Westheimer 1999), or even from a sensory to a motor task (Meegan et al. 2000).

The learning observed in the work described earlier

LEARNING \& MEMORY 10:141-147 @ 2003 by Cold Spring Harbor Laboratory Press ISSN1072-0502/03 \$5.00

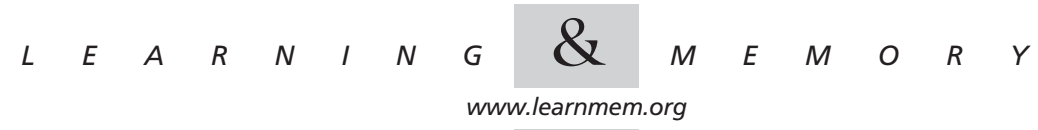


could result from improved timing per se, or an enhanced ability to store and/or compare the standard and comparison stimuli. In the present study, experiments are designed to discriminate between these two hypotheses by using a single stimulus protocol in which the subjects received only a test interval rather than both a test and the standard interval on each trial (see Materials and Methods). Subjects were trained in this manner on one interval and tested to see if the resultant learning generalized to the trained stimulus presented with alternate tone pitches. Additionally, generalization to a perceptually distinct continuous tone with the same duration of the trained interval was also examined. The goal of this design was to increase the subjects' focus on the time span represented by the stimulus and decrease the use of their ability to store and compare stimuli (see Discussion).

Experiments on auditory temporal processing generally train on one particular target stimulus. However, experiments in the visual system have shown that the type of task being performed during training can determine the features over which learning generalizes (Ahissar and Hochstein 1997). Thus the generalization patterns for interval learning may reflect the ease or difficulty of the task. To examine this possibility, naive subjects performed a parallel learning experiment in which they were trained on two intervals simultaneously. In addition to the increased difficulty because of a higher memory load, subjects were forced to attend to the frequency of the stimulus' tones, as it signaled which of the two standards to use for comparison. However, subjects showed significant learning and generalization across frequencies on both trained intervals. Together, the results from our experiments provide evidence for centralized but dedicated, or interval-specific, timing mechanisms.

\section{RESULTS}

\section{Interval Generalization}

We first established that perceptual learning of interval discrimination is observed with the single-stimulus protocol adopted here. Figure 1A shows the average learning curve for 13 subjects trained for $10 \mathrm{~d}$ on the $100-\mathrm{msec}$ interval discrimination task with tones at $1 \mathrm{kHz}$. An orthogonal trend analysis revealed a significant linear component $\left(F_{1,120}=28.7, P<0.001\right)$, indicating that performance improved with training. Because our goal was to examine how perceptual learning generalizes, each subject's learning curve was analyzed independently. All of the individual curves are presented in Figure 1B. Ten of the 13 subjects exhibited significant learning curves as determined by orthogonal linear trend analysis $(P<0.05)$.

To examine transfer of learning, we tested subjects on four different conditions before (pretest) and after training (posttest). Three of the conditions were intervals with the following properties: the trained stimulus of $100 \mathrm{msec}$ at 1

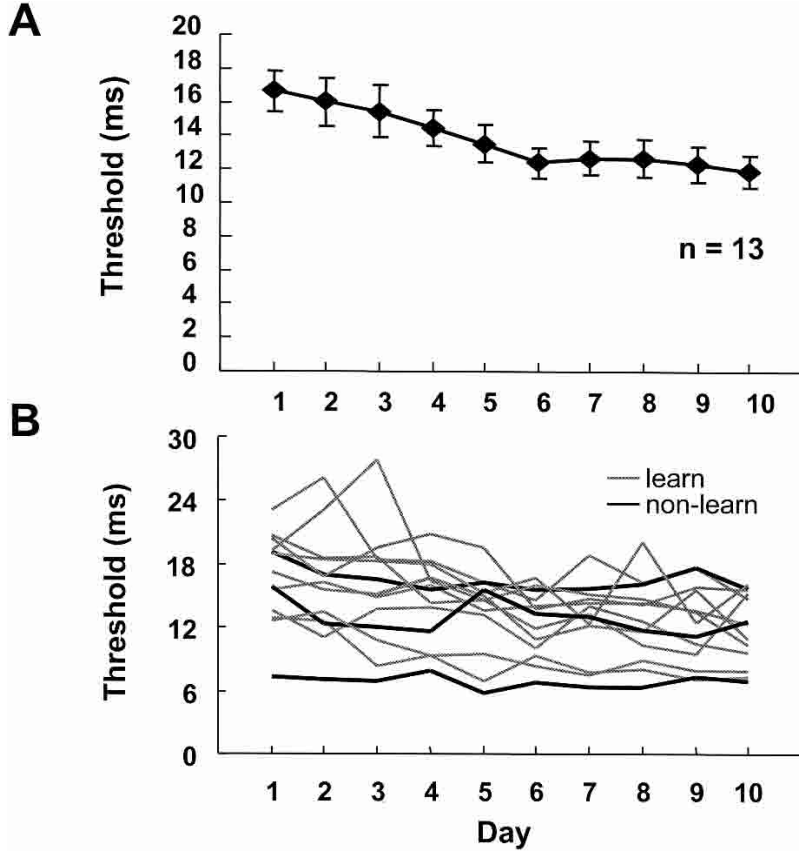

Figure 1 Training on the $100-\mathrm{msec} / 1-\mathrm{kHz}$ task results in perceptual learning. (A) Average learning curve for 13 subjects. Subjects were trained for approximately an hour a day (12 blocks of 60 trials) over $10 \mathrm{~d}$. (B) Individual learning curves for each subject. Subjects that showed significant learning are in gray, nonlearners in black.

$\mathrm{kHz}$, the 100-msec interval at an alternate frequency of 3.75 $\mathrm{kHz}$ tones, and an alternate interval of $200 \mathrm{msec}$ at the trained $1-\mathrm{kHz}$ frequency. The fourth condition, 100-msecdur (100 msec-duration) was based on the trained time span and a $1-\mathrm{kHz}$ frequency but was presented as a single continuous tone so that subjects had to discern changes in tone duration. Subjectively, interval and duration stimuli are perceptually very different. The pretest and posttest data from the 10 subjects that had significant learning curves are shown in Figure 2. A two-way analysis of variance with repeated measures on one factor (pretest $\times$ posttest) revealed a significant effect of training $\left(F_{1,36}=42, P<0.001\right)$ as well as a significant interaction $\left(F_{1,36}=5.4, P<0.005\right)$. Paired $t$-tests revealed a significant difference between the pre- and posttest for all of the conditions except the 200msec/1-kHz interval task. Additionally, there was not a significant difference as determined by $F$-test for effect of trials between the size of the learning effect between the trained and the $100-\mathrm{msec} / 3.75-\mathrm{kHz}$ conditions $\left(F_{1,36}=0.27\right.$, $P<0.61)$ and between the trained and 100 -msec-dur tasks $\left(F_{1,36}=2.86, P=0.10\right)$, indicating complete generalization to the $100-\mathrm{msec} / 3.75-\mathrm{kHz}$ and $100-\mathrm{msec}$ dur tasks.

It is important to determine whether this pattern of generalization is specific to training on $100-\mathrm{msec}$ intervals or is a general feature of interval learning. To verify this issue, we performed a similar set of experiments, except

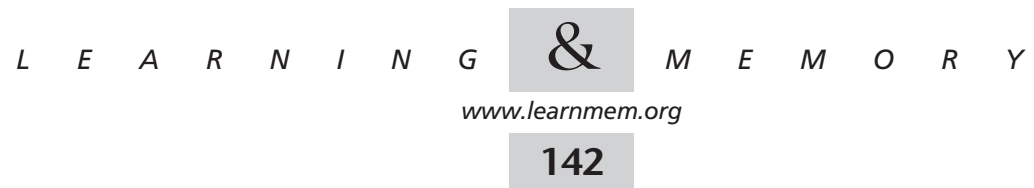




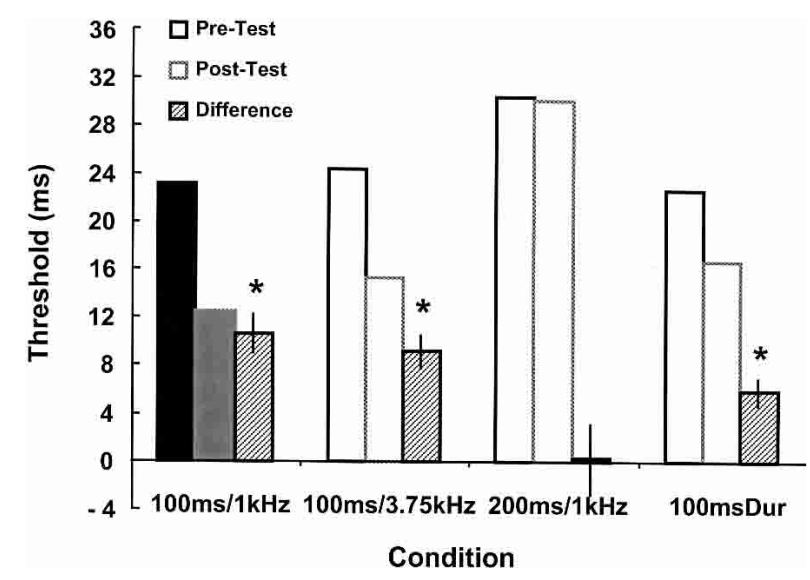

Figure 2 Generalization data from learners trained on the 100$\mathrm{msec} / 1-\mathrm{kHz}$ task. Two days of tests before (pretest) and after (posttest) training were obtained. Solid bars indicate the trained condition. There was a significant decrease in the threshold for the 100msec $/ 1-\mathrm{kHz} \quad(P<0.001), 100-\mathrm{msec} / 3.75-\mathrm{kHz} \quad(P<0.001)$, and 100 -msec-dur $(P<0.001)$ tasks, but not in the $200-\mathrm{msec} / 1-\mathrm{kHz}$ task $(P=0.98)$.

that subjects were trained on an interval of $200 \mathrm{msec}$ with $1-\mathrm{kHz}$ tones. Analogous to the 100-msec generalization experiments, the four test conditions were $200 \mathrm{msec} / 1 \mathrm{kHz}$ (trained condition), $100 \mathrm{msec} / 1 \mathrm{kHz}, 200 \mathrm{msec} / 3.75 \mathrm{kHz}$, and 200-msec dur at $1 \mathrm{kHz}$. Five of the 12 subjects exhibited significant learning curves, indicating that the 200-msec task was a more difficult learning task. The lower percentage of learners may also be due to a floor effect, as many subjects in this group had low pretest threshold values. When the pre- and posttest values of the learners were examined, the results were qualitatively equivalent to those for the 100-msec-based generalization task. A two-way ANOVA with repeated measures on pretest $\times$ posttest showed that training had a significant effect $\left(F_{1,16}=40.32\right.$, $P<0.001)$ as well as a significant interaction $\left(F_{1,16}=3.88\right.$, $P<0.05)$. As shown in Figure 3, learners improved significantly on the trained task as well as on the tasks based on the trained interval, but showed no significant learning on the untrained interval. $F$-tests for differences in learning within groups were not significant between the trained condition and the $200-\mathrm{msec} / 3.75-\mathrm{kHz}$ task $\left(F_{1,16}=1.32\right.$, $P=0.26)$ or for the 200-msec dur condition $\left(F_{1,16}=2.77\right.$, $P=0.12$ ). This is consistent with the results of the 100msec-based experiments, showing that learning on the trained condition generalized completely across frequency and stimulus type.

\section{Parallel Learning Experiments}

These experiments present evidence that improvements in interval discrimination generalize to a duration discrimination task and to interval stimuli presented at untrained frequencies. It is possible, however, that frequency generali- zation was observed because the task did not require the subjects to attend to the frequency of the stimuli. To examine this issue, we trained subjects simultaneously on two different target intervals identified by their frequency. Twenty subjects underwent a testing procedure identical to that of the previous experiments with the following four conditions: $50 \mathrm{msec} / 1 \mathrm{kHz}, 50 \mathrm{msec} / 4 \mathrm{kHz}, 200 \mathrm{msec} / 4$ $\mathrm{kHz}$, and $200 \mathrm{msec} / 1 \mathrm{kHz}$. However, the organization of the training sessions was slightly different. For each block, subjects heard target stimuli for a $50-\mathrm{msec} / 1 \mathrm{k}-\mathrm{Hz}$ interval and then a $200-\mathrm{msec} / 4 \mathrm{kHz}$ interval. After this, they were presented with 120 trials that randomly interleaved stimuli that were either based on the $50-\mathrm{msec} / 1-\mathrm{kHz}$ or $200-\mathrm{msec} /$ $4-\mathrm{kHz}$ conditions. In this experiment, frequency signaled the target interval on which the subjects needed to base their judgment. For example, if the subject received a 100msec stimulus presented with $1-\mathrm{kHz}$ tones, the correct response would be "long", as the lower frequency indicates the subject should base their answer on the 50-msec target. However, "short" would be the correct response if the stimulus used $4-\mathrm{kHz}$ tones, as the correct target would be 200-msec long.

Overall, subjects showed simultaneous learning on both the $50-\mathrm{msec} / 1-\mathrm{kHz}$ and the $200-\mathrm{msec} / 4-\mathrm{kHz}$ training intervals. The average learning curves for all 20 subjects are presented in Figure 4. Both these curves showed a significant orthogonal trend. From the 20 subjects, 11 showed significant learning on both the 50- and 200-msec intervals. There were 6 subjects that showed significant learning on only one of the two intervals (the remaining 3 showed no learning). Five of these 6 improved on the 50-msec interval. Consistent with the 200-msec interval generalization experiments, the 200-msec stimuli may be more difficult to learn. It is also possible that these subjects chose to pay less

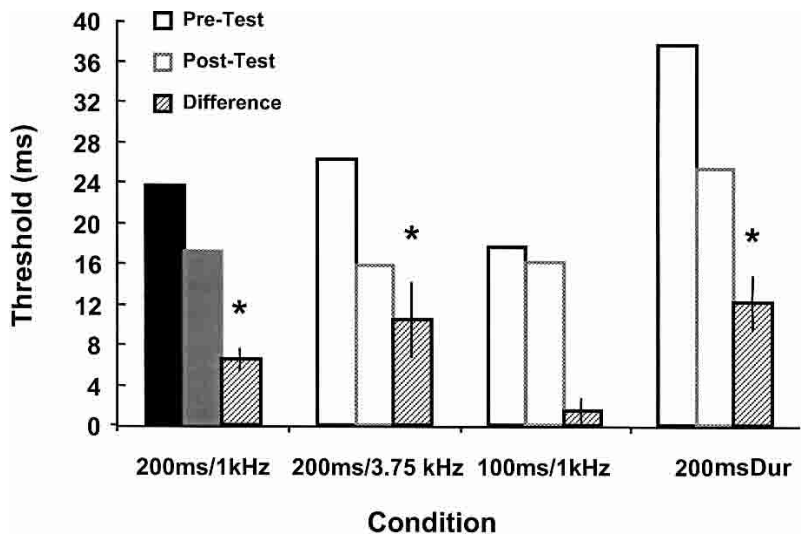

Figure 3 Generalization data from learners trained on the 200msec/1-kHz task. Solid bars indicate the trained condition. A significant decrease from pre- to posttest threshold was seen in the $200-\mathrm{msec} / 1-\mathrm{kHz}(P<0.01), 200-\mathrm{msec} / 3.75-\mathrm{kHz}(P<0.05)$, and 200 -msec-dur conditions as measured by paired $t$-test. There was no significant decrease in the $100-\mathrm{msec} / 1-\mathrm{kHz}$ task $(P=0.25)$.

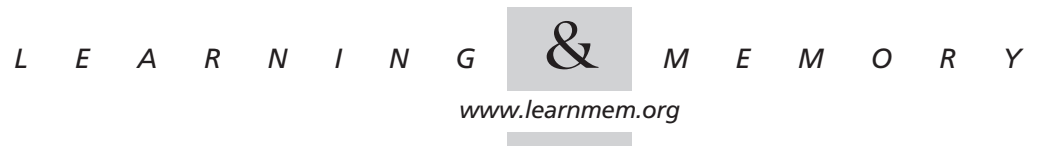




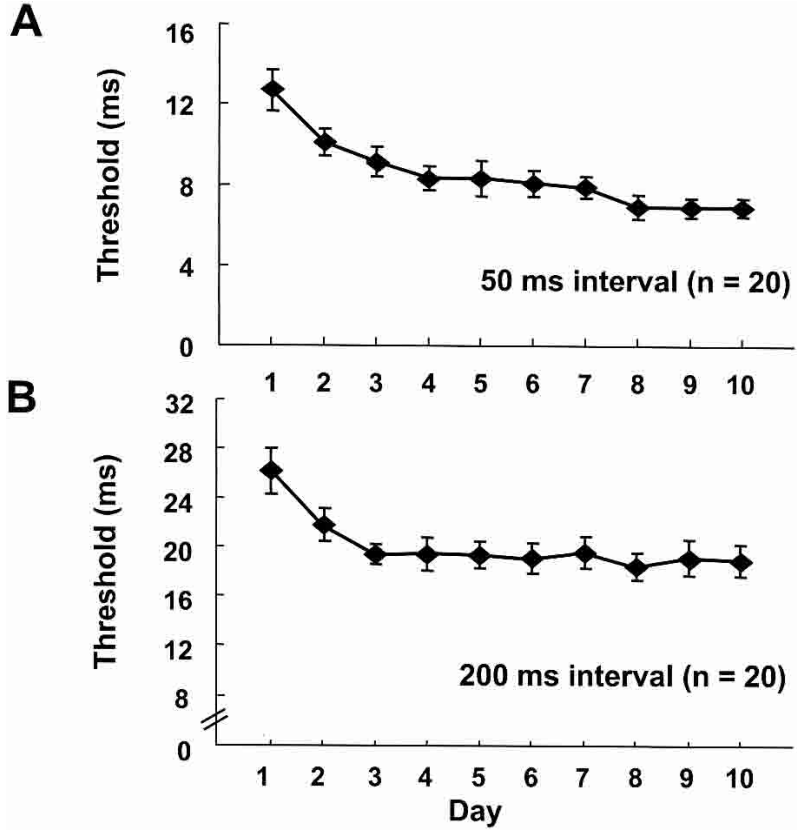

Figure 4 Parallel learning on two different conditions. (A) Average learning curve for the $50-\mathrm{msec} / 1-\mathrm{kHz}$ interval for subjects. The curve shows significant learning as measured by orthogonal trend analysis $\left(F_{1,190}=49.63, P<0.001\right)$. (B) Learning curve for the 200$\mathrm{msec} / 4-\mathrm{kHz}$ interval. The average learning is significant $\left(F_{1,190}=14.02, P<0.001\right)$. Subjects were trained on the $50-\mathrm{msec}$ and 200-msec intervals simultaneously for $10 \mathrm{~d}$.

attention to one type of stimuli when performing the task. As shown in Figure 5, the pre- and posttest data for the 11 subjects that learned on both intervals revealed a significant effect of training $\left(F_{1,40}=124, P<0.001\right)$. However, the interaction was not significant $\left(F_{1,40}=1.31, P=0.28\right)$, indicating that there was an improvement in performance across all of the conditions. These learners improved significantly on the trained conditions, but also on the untrained conditions, thus showing complete spectral generalization despite the importance of frequency in this task.

\section{DISCUSSION}

The results presented here show that temporal perception, as measured by interval discrimination, undergoes learning. This form of perceptual learning is specific to the time of the trained interval, but generalizes from an interval to duration (filled interval) task, as well as to novel frequencies. The transfer of learning to novel frequencies is consistent with previous reports in which a two-interval, forcedchoice paradigm was used (Wright et al. 1997; Nagarajan et al. 1998; Westheimer 1999).

Many experiments have examined perceptual learning in the visual system and whether it generalizes across various stimulus features. These studies have shown high de- grees of both task and retinotopic specificity. For example, spatial discrimination learning on pop-out, vernier, and bisection tasks generally does not transfer to different stimuli orientations or to untrained retinal quadrants (Karni and Sagi 1991; Fahle et al. 1995; Crist et al. 1997). These psychophysical data have been of value in helping to narrow down the neural basis of visual learning. They indicate that learning may be the result of changes occurring in V1, because V1 is the first site in which orientation-selective neurons are found, and these cells still have small receptive fields (Gilbert et al. 2001). Adini et al. (2002) have proposed a mechanism for contrast perception learning in visual cortex based on a model of plasticity consistent with their psychophysical data. More directly, a study of V1 after perceptual learning specific to both stimulus orientation and location found a correlated cellular change (Schoups et al. 2000). In this study, plasticity in the tuning of a subgroup of V1 cells related to the trained orientation was proposed to underlie perceptual learning. Another study has failed to observe such electrophysiological changes; however, the behavioral results were also different, as the learning that was seen generalized across locations (Ghose et al. 2002).

In contrast with visual perceptual learning, few data are available on temporal perceptual learning in the auditory system. Specifically, it is not known whether the neural loci for timing are distributed throughout multiple areas or centralized to specific structures (Ivry 1996; Buonomano and Karmarkar 2002). The cerebellum, which contributes to the timing of motor responses (Ivry 1993; Perrett et al. 1993; Medina et al. 2000), has also been implicated in the timing of sensory events (Ivry and Keele 1989; Ivry 1993; Jueptner et al. 1995). Others have presented evidence that timing in the range of hundreds of milliseconds is localized in basal ganglia (Artieda et al. 1992; Harrington et al. 1998a) or cortical areas (Harrington et al. 1998b; Onoe et al. 2001).

Although the current study does not directly address the localization of timing, it, too, can offer insights based on its generalization profiles and the known functional anatomy of the auditory system. However, it is important to establish that the observed learning is actually due to an improvement in temporal resolution. Learning in previous experiments could have been due to an overall enhancement in the ability to store and compare stimuli. Assuming that the focus of this strategy would be the temporal difference between the interval stimuli for comparison, it is compatible with generalization to novel frequencies and not to novel intervals. The current experiments decreased the likelihood of this strategy in two ways. The first was to remove the standard interval from the trials in both testing and training, thus eliminating trial-by-trial comparison of the target and standard interval. The design of this experiment, therefore, encouraged subjects to form a dynamic representation of the time frame involved, and to adjust this timing based on the feedback from their decisions. Explicit memo-

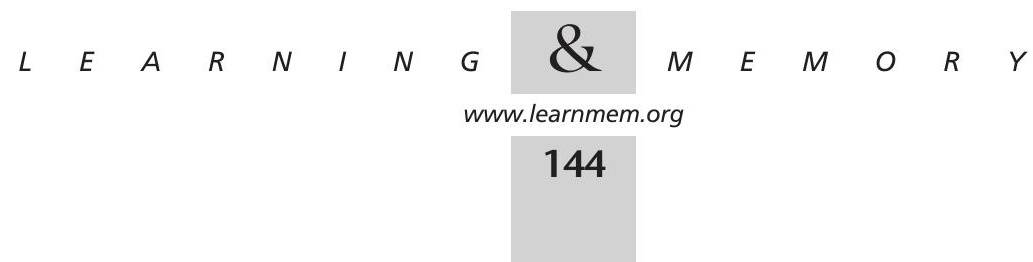




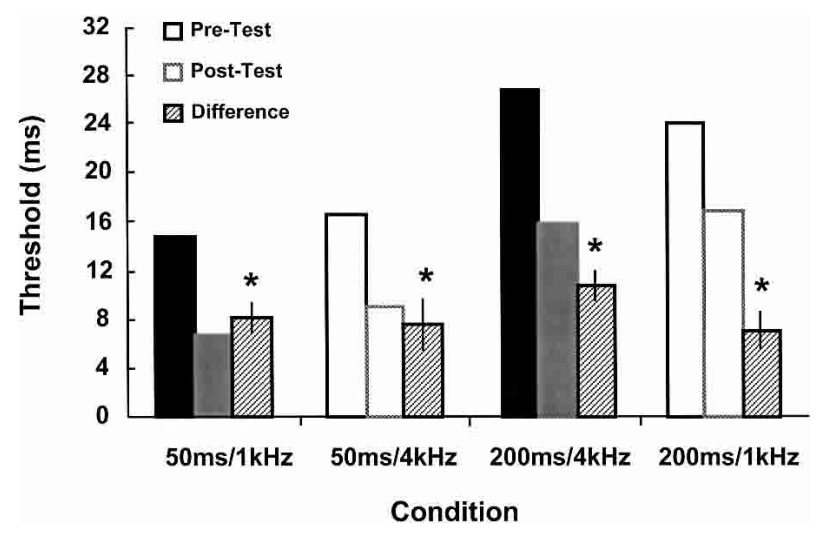

Figure 5 Generalization data for the learners in the parallel learning experiments. Trained conditions are indicated by solid bars, and show a significant decrease in threshold as measured by paired $t$-tests $(P<0.001$ for both). A significant improvement in performance is seen in the $50-\mathrm{msec} / 4-\mathrm{kHz}(P<0.01)$ and $200-\mathrm{msec} / 1-$ $\mathrm{kHz}(P<0.001)$ tasks.

rization of the standard stimulus is not possible during the trials, and subjects reported listening to the standard only one to five times before the trial block. We found that subjects still showed statistically significant improvements in their discrimination thresholds after training with this protocol, indicating that the underlying cause for learning was an improvement in the actual resolution of the mechanisms underlying timing.

The second aspect of our approach was to examine generalization to a duration discrimination task. Perceptually, the duration and interval stimuli are very different. Indeed, previous research has shown that duration and interval discrimination thresholds differ significantly, which implies that they may represent separate tasks (Rammsayer and Lima 1991; Grondin et al. 1998). Thus, it seems likely that improvement in the ability to store or compare stimuli should not generalize to a duration task. Because we observed generalization from the interval to duration task, our results indicate that learning relied on changes in temporal processing.

The focus of this study is on the specificity of timing mechanisms, and that is determined here by the generalization profile of training-dependent learning. It is possible that significant learning is taking place during the pretest; however, this learning may be confounded with improvements because of increased familiarity with the experimental setup. It is also impossible to separate the contribution of any particular stimulus condition to learning in data from the pretest. By using data from subjects that showed significant improvement as a result of training, we can examine the generalization produced specifically by temporal learning on the trained stimulus.

The generalization to novel frequencies, but not to novel intervals, in these experiments indicates that timing for any given interval relies on centralized circuits that are accessed across different channels. However, it is possible that independent timing mechanisms exist for each frequency, but if a task does not require attending to a given frequency, all the circuits can undergo learning through top-down mechanisms. Experiments in the visual system have shown that the type of task being performed during training can determine the features over which learning generalizes. For example, Ahissar and Hochstein (1997) have shown that increasing task difficulty by including a larger training stimulus set can decrease generalization. Thus the generalization seen in interval learning may also reflect the difficulty of the task, or more generally the salient features of the task. The parallel learning experiments presented here indicate that this is not the case. This task was more difficult, as two different intervals were learned simultaneously and it required attention to frequency information. Because generalization was still observed to novel frequencies, these data indicate that this pattern of generalization is a general feature of temporal perceptual learning.

The specificity for spatial location of perceptual learning in the visual system is generally taken as evidence that learning relies on retinotopic changes in V1 (Gilbert et al. 2001). Our observation that temporal perceptual learning in the auditory system generalizes to untrained stimulus frequencies indicates that timing mechanisms may not be located in tonotopically organized structures such as A1, because most reported receptive fields there do not respond to both 1 and $4 \mathrm{kHz}$ (Phillips and Irvine 1981; Recanzone et al. 2000). However, it should be noted that some primary auditory cortex cells, in cats, for example, can have broad, even multipeaked tuning curves (Sutter and Schreiner 1991), making it physiologically possible for A1 neurons to account for frequency generalization.

Auditory learning also transferred from an interval to a duration task based on the same time span. This diverges from visual psychophysics results, in which spatial learning on tasks such as bisection discrimination does not generalize to even a vernier task that involves the same visual stimuli (Crist et al. 1997). Overall, although most visual learning is specific to several visual stimulus features, the perceptual learning in the auditory experiments presented here is specific only to the temporal domain. Because the characteristics of most subcortical auditory neurons as described in animal preparations (Kiang et al. 1965; Godfrey et al. 1975a,b; Nuding et al. 1999) indicate that they would respond differently to unfilled (interval) and filled (duration) stimuli, it is unlikely that temporal learning relies on early subcortical plasticity mechanisms. Rather, our data indicate that learning is occurring at later, less dedicated stages of auditory processing, where interval and duration stimuli have common integration pathways. These sites would include cortical areas and cerebellar circuits receiving cortical input.

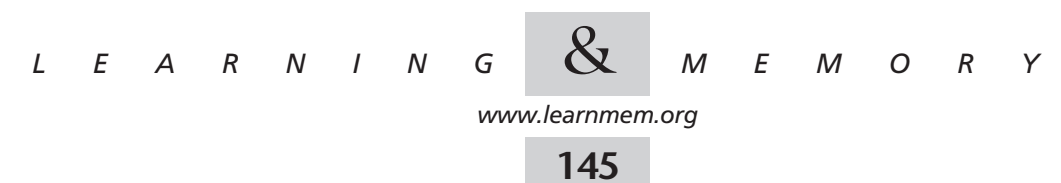




\section{MATERIALS AND METHODS}

\section{Experimental Subjects}

A total of 45 volunteers between the ages of 18 and $30 \mathrm{yr}$ with normal hearing participated in one of the three types of experiments described as follows. Subjects performed the experiment at the same time daily (with the exception of weekends) for $14 \mathrm{~d}$. The subjects had no previous experience in this type of discrimination task and were paid for their participation.

\section{Protocol}

A single stimulus protocol was used for all experiments. At the beginning of the trial block for a particular condition, subjects were presented with a target stimulus of two tones of a specific frequency separated by a fixed interval (T). Participants could choose to listen to this target as many times as they wished. After this stage, only test stimuli of a single pair of tones that were either shorter or longer than the target stimulus $(\mathrm{T} \pm \Delta \mathrm{t})$ were presented. Subjects made a forced-choice decision as to whether the trial stimulus seemed shorter or longer than the target interval by pressing one of two buttons on a computer mouse. They were provided with immediate visual feedback. All stimuli were generated in Matlab and presented through Optimus Pro 40 headphones. Tones were 15 msec long including a $5 \mathrm{msec}$ on-and-off ramp.

All experiments consisted of $2 \mathrm{~d}$ of pretest, $10 \mathrm{~d}$ of a training phase, and $2 \mathrm{~d}$ of posttest. For the pre- and posttest, subjects completed the experimental task for four test conditions (see following), which were presented in a random order. Training was done for $10 \mathrm{~d}$ on a specified subset of the test conditions.

\section{0-msec and 200-msec Interval Generalization Experiments}

Independent experiments were run in which subjects were trained on either a 100- or 200-msec target interval. Each session, whether on a testing or training day, consisted of 12 blocks of 60 trials each. On pre- and posttest days, the subjects ran each of the four test conditions a total of three times. For the 100-msec-based tasks, the testing conditions were three types of interval targets: $100 \mathrm{msec} / 1 \mathrm{kHz}$ tones, $100 \mathrm{msec} / 3.75 \mathrm{kHz}$, and $200 \mathrm{msec} / 1 \mathrm{kHz}$. The fourth condition was a duration-based task in which the target was a single $1-\mathrm{kHz}$ tone with a $100-\mathrm{msec}$ duration. For the 10 training days, subjects received 12 blocks on the target condition $(100 \mathrm{msec} / 1 \mathrm{kHz})$.

To determine that results were a general property of interval learning, rather than specific to the $100-\mathrm{msec}$ target interval, a separate group was trained with a $200-\mathrm{msec} / 1-\mathrm{kHz}$ target stimulus. In these experiments, the test conditions were $100 \mathrm{msec} / 1 \mathrm{kHz}$, $200 \mathrm{msec} / 1 \mathrm{kHz}, 200 \mathrm{msec} / 3.75 \mathrm{kHz}$, and a $200-\mathrm{msec} / 1-\mathrm{kHz}$ duration target.

\section{Parallel Interval Learning}

In these experiments, subjects were trained on two stimulus conditions concurrently. During pre- and posttesting the following four interval conditions were used: $50 \mathrm{msec} / 1 \mathrm{kHz}, 50 \mathrm{msec} / 4 \mathrm{kHz}, 200$ $\mathrm{msec} / 1 \mathrm{kHz}$, and $200 \mathrm{msec} / 4 \mathrm{kHz}$. These were each tested in blocks of 60 trials twice in random order on the two pretest and two posttest days. Subjects were trained on two intervals simultaneously. Training was done on seven blocks of 120 trials with trial stimuli based on $50-\mathrm{msec} / 1-\mathrm{kHz}$ and $200-\mathrm{msec} / 4-\mathrm{kHz}$ targets randomly interleaved within the blocks.

\section{Analysis and Determination of Threshold}

Threshold values were determined in the manner described previously (Wright et al. 1997). In brief, an adaptive procedure was used during each block, in which $\Delta \mathrm{t}$ was decreased after three correct answers, and increased after one incorrect answer. The $\Delta t$ values at points that reversed from increasing to decreasing or vice versa were recorded. Because the stimuli in our protocols had an interval of $\mathrm{T} \pm \Delta \mathrm{t}$, we defined threshold as 2 multiplied by the mean of all reversal values excluding the first three. Significance of the learning curves was determined by linear orthogonal trend analysis (Bruning and Kintz 1987).

\section{ACKNOWLEDGMENTS}

The authors would like to thank Dr. Steven Engel for comments on earlier versions of this manuscript. This research was supported by the EJLB Foundation, the DoD (NDSEG), and by the NSF.

The publication costs of this article were defrayed in part by payment of page charges. This article must therefore be hereby marked "advertisement" in accordance with 18 USC section 1734 solely to indicate this fact.

\section{REFERENCES}

Adini, A., Sagi, D., and Tsodyks, M. 2002. Context-enabled learning in the human visual system. Nature 415: 790-793.

Ahissar, M. and Hochstein, S. 1997. Task difficulty and the specificity of perceptual learning. Nature 387: 401-406.

Artieda, J., Pastor, M.A., Lacruz, F., and Obeso, J.A. 1992. Temporal discrimination is abnormal in Parkinson's disease. Brain 115: 199-210.

Bruning, J.L. and Kintz, B.L. 1987. Computational handbook of statistics. 3rd ed., pp. 145-158. Scott, Foresman and Company, Glenview, IL.

Buonomano, D.V. and Karmarkar, U.R. 2002. How do we tell time? Neuroscientist 8: 42-51.

Carr, C.E. 1993. Processing of temporal information in the brain. Annu. Rev. Neurosci. 16: 223-243.

Crist, R.E., Kapadia, M.K., Westheimer, G., and Gilbert, C.D. 1997. Perceptual learning of spatial localization: Specifiy for orientation, position, and context. J. Neurophysiol. 78: 2889-2894.

Doupe, A.J. and Kuhl, P.K. 1999. Birdsong and human speech: Common themes and mechanisms. Annu. Rev. Neurosci. 22: 567-631.

Fahle, M., Edelman, S., and Poggio, T. 1995. Fast perceptual learning in hyperacuity. Vision Res. 35: 3003-3013.

Ghose, G.M., Yang, T., and Maunsell, J.H.R. 2002. Physiological correlates of perceptual learning in monkey V1 and V2. J. Neurophysiol. 87: $1867-1888$

Gibbon, J., Malapani, C., Dale, C.L., and Gallistel, C.R. 1997. Toward a neurobiology of temporal cognition: Advances and challenges. Curr. Opin. Neurobiol. 7: 170-184.

Gilbert, C.D., Sigman, M., and Crist, R.E. 2001. The neural basis of perceptual learning. Neuron 31: 681-697.

Godfrey, D.A., Kiang, N.Y.S., and Norris, B.E. 1975a. Single unit activity in the posteroventral cochlear nucleus of the cat. J. Comp. Neurol. 162: $247-268$

. 1975b. Single unity activity in the dorsal cochlear nucleus of the cat. J. Comp. Neurol. 162: 269-284

Grondin, S., Meilleur-Wells, G., Ouellette, C., and Macar, F. 1998. Sensory effects on judgments of short time-intervals. Psychol. Res. 61: 261-268.

Harrington, D.L., Haaland, K.Y., and Hermanowicz, N. 1998a. Temporal processing in the basal ganglia. Neuropsychology 12: 3-12.

Harrington, D.L., Haaland, K.Y., and Knight, R.T. 1998b. Cortical networks underlying mechanisms of time perception. J. Neurosci. 18: $1085-1095$.

Ivry, R. 1993. Cerebellar involvement in the explicit representation of temporal information. Ann. N.Y. Acad. Sci. 682: 214-230. - 1996. The representation of temporal information in perception and motor control. Curr. Opin. Neurobiol. 6: 851-857.

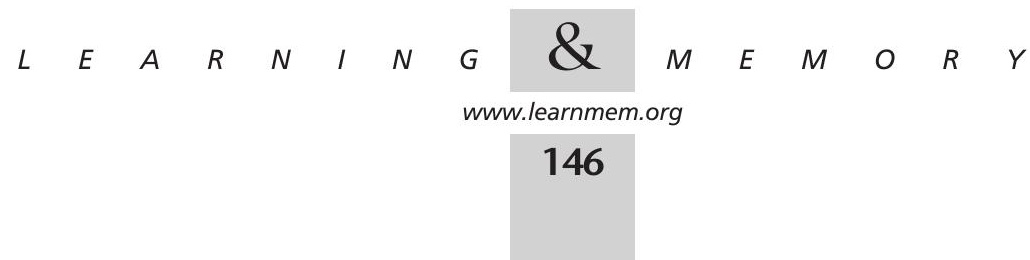


Ivry, R. and Keele, S.W. 1989. Timing functions of the cerebellum. J. Cogn. Neurosci. 1: 136-152.

Jeffress, L.A. 1948. A theory of sound localization. J. Comp. Physiol. Psychol. 41: 35-59.

Jueptner, M., Rijntjes, C., Weiller, C., Faiss, J.H., Timmann, D., Mueller, S.P., and Diener, H.C. 1995. Location of a cerebellar timing process using PET. Neurology 45: 1540-1545.

Karni, A. and Sagi, D. 1991. Where practice makes perfect in texture discrimination: Evidence for primary visual cortex plasticity. Proc. Natl. Acad. Sci. 88: 4966-4970.

Kiang, N.Y.S., Pfeiffer, R.R., Warr, W.B., and Backus, A.S.N. 1965. Stimulus coding in the cochlear nucleus. Trans. Am. Otol. Soc. 53: 35-58.

Medina, J.F., Garcia, K.S., Nores, W.L., Taylor, N.M., and Mauk, M.D. 2000 Timing mechanisms in the cerebellum: Testing predictions of a large-scale computer simulation. J. Neurosci. 20: 5516-5525.

Meegan, D.V., Aslin, R.N., and Jacobs R.A. 2000. Motor timing learned without motor training. Nat. Neurosci. 3: 860-862.

Merzenich, M.M., Jenkins, W.M., Johnston, P., Schreiner, C., Miller, S.L., and Tallal, P. 1996. Temporal processing deficits of language-learning impaired children ameliorated by training. Science 271: 77-81.

Nagarajan, S.S., Blake, D.T., Wright, B.A., Byl, N., and Merzenich, M.M. 1998. Practice-related improvements in somatosensory interval discrimination are temporally specific but generalize across skin location, hemisphere, and modality. J. Neurosci. 18: 1559-1570.

Nuding, S.C., Chen, G.-D., and Sinex, D.G. 1999. Monaural response properties of single neurons in the chinchilla inferior colliculus. Hear. Res. 131: 89-106.

Onoe, H., Komori, M., Onoe, K., Takechi, H., Tsukada, H., and Watanabe, Y. 2001. Cortical networks recruited for time perception: A monkey positron emission tomography (PET) study. Neuroimage 13: 37-45.

Perrett, S.P., Ruiz, B.P., and Mauk, M.D. 1993. Cerebellar cortex lesions disrupt learning-dependent timing of conditioned eyelid responses. $J$. Neurosci. 13: 1708-1718.
Phillips, D.P. and Irvine, D.R.F. 1981. Responses of single neurons in physiologically defined primary auditory cortex (AI) of the cat: Frequency tuning and responses to intensity. J. Neurophysiol. 45: 48-58.

Rammsayer, T.H. and Lima, S.D. 1991. Duration discrimination of filled and empty auditory intervals: Cognitive and perceptual factors. Percept. Psychophys. 50: 565-574.

Recanzone, G.H., Guard, D.C., and Phan, M.L. 2000. Frequency and intensity response properties of single neurons in the auditory cortex of the behaving macaque monkey. J. Neurophysiol. 83: 2315-2331.

Schoups, S., Vogels, R., Quian, N., and Orban, G. 2000. Practising orientation identification improves orientation coding in V1 neurons. Nature 412: 549-553.

Shannon, R.V., Zeng, F.G., Kamath, V., Wygonski, J., and Ekelid, M. 1995. Speech recognition with primarily temporal cues. Science 270: 303-304.

Sutter, M.L. and Schreiner, C.E. 1991. Physiology and topography of neurons with multipeaked tuning curves in cat primary auditory cortex. J. Neurophysiol. 65: 1207-1226.

Tallal, P., Miller, S.L., Bedi, G., Byma, G., Wang, X., Nagarajan, S.S., Schreiner, C., Jenkins, W.M., and Merzenich, M.M. 1996. Language comprehension in language-learning impaired children improved with acoustically modified speech. Science 271: 81-84.

Westheimer G. 1999. Discrimination of short time intervals by the human observer. Exp. Brain Res. 129: 121-126.

Wright, B.A., Buonomano, D.V., Mahncke, H.W., and Merzenich, M.M. 1997. Learning and generalization of auditory temporal-interval discrimination in humans. J. Neurosci. 17: 3956-3963.

Received September 16, 2002; accepted in revised form January 16, 2003 .

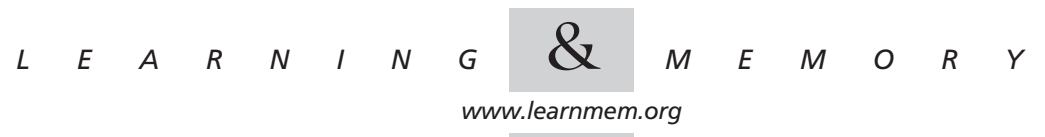




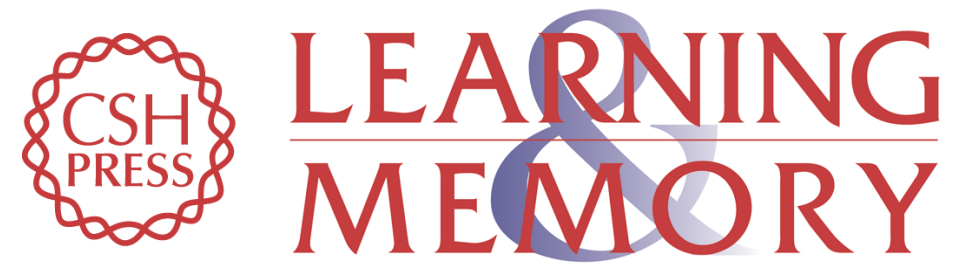

\section{Temporal Specificity of Perceptual Learning in an Auditory Discrimination Task}

Uma R. Karmarkar and Dean V. Buonomano

Learn. Mem. 2003, 10:

Access the most recent version at doi:10.1101//m.55503

References This article cites 38 articles, 10 of which can be accessed free at: http://learnmem.cshlp.org/content/10/2/141.full.html\#ref-list-1

License

Email Alerting Receive free email alerts when new articles cite this article - sign up in the box at the Service top right corner of the article or click here. 